\title{
Resistance to chemotherapy and anti-angiogenic therapy in ovarian cancer
}

\author{
Verena Wieser (D) Christian Marth
}

Received: 22 October 2018 / Accepted: 6 February 2019 / Published online: 21 February 2019

(C) The Author(s) 2019

\begin{abstract}
Summary Ovarian cancer (OC) is the foremost lethal gynaecologic malignancy and among the top five deadliest cancers in women. Current treatment comprises a combination therapy of surgery, platinumbased chemotherapy and anti-vascular endothelial growth factor (VEGF) antibodies. However, patients typically experience a disease relapse within two years. Recurrent OC is incurable and resistance to platins and anti-VEGF treatment is a major determinant of prognosis. Understanding the molecular mechanisms that contribute to tumour metastasis and chemoresistance are essential to improve patient outcome and especially survival. In a current OC model, tumour metastasis and chemoresistance critically depend on the biology of cancer stem cells (CSCs). Recent studies also suggest that intratumour heterogeneity is the main cause of treatment failure due to chemoresistance. Furthermore, the proinflammatory tumour microenvironment seems to contribute to metastasis and chemoresistance. Despite an improved understanding of the complex interplay between classical mechanisms of drug inactivation or efflux, clonal selection and the tumour microenvironment, mechanisms of resistance in human OC are poorly understood. This review summarises current concepts in the treatment of OC, mechanisms of resistance to chemotherapy and angiogenic inhibitors and approaches to overcome drug resistance.
\end{abstract}

Keywords Ovarian cancer - Chemoresistance · Platinum resistance $\cdot$ Angiogenic inhibitors · VEGF

V. Wieser, MD, $\mathrm{PhD}(\bowtie) \cdot$ C. Marth, MD, $\mathrm{PhD}$

Department of Obstetrics and Gynaecology, Medical

University of Innsbruck, Anichstraße 35, 6020 Innsbruck, Austria

verena.wieser@i-med.ac.at

\section{Introduction}

The frontline therapy of ovarian cancer (OC) consists of surgery and platinum-based chemotherapy (usually carboplatin area under the curve [AUC] 5-6 and paclitaxel $175 \mathrm{mg} / \mathrm{m}^{2}$ every 3 weeks for six cycles as demonstrated in the GOG-158 study) [1]. Patients with advanced disease (i.e. FIGO IIIb and higher) additionally receive anti-angiogenic therapy during chemotherapy (GOG-218: bevacizumab $15 \mathrm{mg} / \mathrm{kg}$ from second cycle carboplatin/paclitaxel; hazard ratio (HR) for progression or death 0.908 [95\% confidence interval (CI), 0.795 to $1.040 ; P=0.16])$ and for maintenance (16 cycles after chemotherapy; HR 0.717 [95\% CI, 0.625 to $0.824 ; P<0.001$ ]) [2]. Although response rates and complete responses after first-line treatment of advanced disease are $>80 \%$ and $40-60 \%$, respectively, most patients will relapse with a median progression-free survival (PFS) of 18 months [3]. In general, patients who respond to primary treatment and relapse within 6 months are considered "platinum-resistant", and patients who relapse more than 6 months after completion of initial therapy are characterized as "platinum-sensitive" [4]. Interestingly, most of "platinum-sensitive" patients will respond to further platinum-based chemotherapy with response rates ranging from 30 to $90 \%$ [5]. In contrast, "platinum-resistant" patients typically have low response rates $(15 \%)$ to subsequent chemotherapy and the outcome of these patients is poor with a median survival not exceeding 12 months [5]. Primary "platinumrefractory" OC patients (those that progress during treatment) are quite uncommon and usually seen with non-serous ovarian cancers such as clear cell or mucinous cancers rather than the more common high grade serous ovarian cancer (HGSOC). "Platinum-refractory" patients also exhibit a poor clinical outcome with comparable low median survival. 
Tumour angiogenesis is an essential process of cancer growth and metastasis and influences the progression of ovarian cancer [6]. Vascular endothelial growth factor (VEGF) is an important promoter of the formation of new blood vessels that contribute to "feeding cancer" [6]. The expression of VEGF and its receptors in ovarian tumours directly correlate with poor prognosis, suggesting that angiogenesis, possibly mediated at least in part by VEGF, influences disease progression $[7,8]$. To date, anti-angiogenic therapy has been identified as one of the most promising targeted therapies in OC. Bevacizumab, a recombinant, humanized, monoclonal VEGF antibody, has been implemented in the first-line treatment with a platinumbased chemotherapy but also in platinum-sensitive (OCEANS trial) and resistant (AURELIA trial) recurrent OC $[2,9,10]$. More specifically, in platinumsensitive recurrent OC gemcitabine and carboplatin plus bevacizumab followed by bevacizumab until progression resulted in a better PFS compared with chemotherapy plus placebo (HR 0.484; 95\% CI, 0.388 to $0.605 ; P<0.0001)$ [9]. Combining bevacizumab with chemotherapy in platinum-resistant OC also improved PFS (HR 0.48; 95\% CI, 0.38 to $0.60 ; P<0.001$ ) [10].

Influences on platinum sensitivity and mechanisms of platinum resistance

Different tumour-specific factors such as histological subtype, clonal selection, tumour mutations and microenvironment but also pharmacokinetic factors influence response and resistance to chemotherapy and anti-angiogenesis [11]. Platinum sensitivity and resistance is well known in patients with HGSOC. Endometrioid, clear cell, mucinous and lowgrade serous OC are less common histotypes and differ from HGSOC in clinical course, tumour mutations, molecular aberrations and in response to chemotherapy. Mucinous, clear cell and low-grade serous cancers tend to be resistant to standard-ofcare [12]. However, patients are still treated with platinum-based chemotherapy first-line due to the lack of proven alternatives. Since mucinous OC shares several common pathological and molecular features with gastrointestinal tumours, it has long been hypothesized that standard gastrointestinal treatments could be more effective for this histotype than the current standard-of-care. In all the phase I/II cohorts of platinum-refractory OC patients treated with some of these approaches (e.g., capecitabine, oxaliplatin, FOLFOX, gemcitabine + oxaliplatin), only a very small number of mucinous OC patients were included, meaning it was difficult to draw clear conclusions [13].

Germ-line mutations in BRCA1 and BRCA2 are wellknown risk factors for developing HGSOC [14]. OC patients with germline (BReast CAncer) BRCA mutations exhibit a favourable outcome and higher responsive- ness to platinum-based therapies [15-17]. However, many sporadic HGSOCs exhibit phenotypic characteristics of germline BRCA mutated tumours. This socalled BRCAness can be defined as a defect in homologous recombination repair and can be, for example, caused by somatic mutation of $B R C A 1 / 2$, epigenetic hypermethylation of the $B R C A 1$ promoter, amplification of EMSY (also known as C11orf30) resulting in BRCA2 silencing, and loss of function mutations of the Fanconi anaemia complementation group family of genes [18]. Importantly, a BRCAness gene expression profile was shown to predict platinum responsiveness [19].

The cytotoxic effect of platins relies on single or double strand DNA breaks and may also cause mitochondrial damage and in turn cell death [19]. Platinum resistance may stem from reduced platinum uptake into the cell or increased efflux evoked by alterations of transport proteins. An increase in DNA repair by alterations of repair proteins such as nucleotide excision repair, mismatch repair, homologous recombination or base excision repair is also classically associated with platinum resistance. These various mechanisms may already exist at diagnosis or are acquired over time [12].

OC stem cells (OCSC) seem to play a potential role in OC recurrence following chemotherapy. Cancer stem cells typically exhibit a slow cycling rate which makes them inherently resistant to standard chemotherapy which, by definition, targets actively proliferating cells [20]. However, the underlying mechanisms that regulate the chemoresistance of OCSCs remain unclear [21]. Kryczek et al. and Silva et al. defined OSCSs via the presence of aldehyde dehydrogenase (ALDH) and CD133 [22, 23]. Furthermore, the presence of ALDH and CD133 positive cells in debulked primary tumour specimen correlated with reduced PFS and overall survival (OS) in ovarian cancer patients [23]. This may be because of the association of high ALDH1Al expression/activity with platinum-resistant cells in vitro. In an in vivo orthotopic mouse model of ovarian cancer, ALDH1A1 silencing sensitized both taxane- and platinum-resistant tumours to chemotherapy [24]. Reimer et al. demonstrated that truncated isoform Vav3.1 is highly expressed in OCSCs and clinically relevant in predicting prognosis and platinum-response as Vav3.1 may be decisively involved in mechanisms causing genuine multidrug resistance [25]. In contrast, in the environment-mediated drug resistance (EMDR) model, cancer cells interact with their surrounding microenvironment and enter a quiescent state due to the complex interplay between tumour and its microenvironment. These surviving populations, which may or may not be OCSCs, can contribute to cancer relapse [26].

Patients who have an initial response to platinum-based chemotherapy are believed to have tumours with intratumour heterogeneity of both in- 
trinsically platinum-resistant cells and also sensitive cells. The sensitive cells undergo apoptosis following chemotherapy (tumour response) but the resistant subpopulation of cells persist and expand, leading to early recurrence in "platinum-resistant" disease. "Platinum-sensitive" patients may respond to platinum, due to the regrowth of the sensitive population. Ultimately however, the "sensitive" cells may alter or mutate, rendering them resistant, or the resistant cell population will outgrow the sensitive population [5].

\section{Mechanisms of anti-VEGF resistance}

Evidence suggests that mechanisms of resistance to anti-VEGF therapy might be mediated by tumour cells and by members of the tumour microenvironment $[11,27,28]$. Tumour hypoxia is a major molecular controller of an "angiogenic switch" that determines a time-restricted event during tumour progression in which the balance between pro- and anti-angiogenic factors tilts towards a pro-angiogenic outcome $[27,29$, 30]. Blocking the VEGF pathway inhibits vessel formation but also promotes recruitment of vascular progenitors and vascular modulators such as tumour-associated macrophages (TAMs), immature monocytes and hemangiocytes. Growing evidence indicates that inflammation controls angiogenesis as infiltrating tumour-associated macrophages have been linked to the escape from anti-angiogenic therapy [29]. Further, M2 polarized TAMs promote tumour vascularization by producing proangiogenic factors and growth factors, including transforming growth factor (TGF$\beta$ ) and VEGF, and attracting leukocytes to further enhance angiogenesis [31].

Recent genomic interrogation of large numbers of HGSOC samples indicated high complexity in terms of genetic aberrations, intra- and intertumor heterogeneity and underscored their lack of targetable oncogenic mutations [32-34]. Subclassifications of HGSOC based on expression profiles, termed "differentiated", "immunoreactive", "mesenchymal" and "proliferative", were shown to have prognostic value. Proliferative and mesenchymal subtypes exhibit poorest survival but derive a comparably greater benefit from treatment with bevacizumab [35].

\section{Concepts to overcome drug resistance}

The response to cytotoxic chemotherapy remains the essential determinant of OC prognosis [36]. The lack of a detailed understanding of the mechanisms that underlie clinical drug resistance has not deterred investigators from initiating a range of clinical trials that aim to tackle the problem. Novel approaches include disruption of homologous recombination (HR) (i.e. poly-ADP-ribose-polymerase [PARP] inhibitors), reversing inflammation or tumour immune escape and simultaneous targeting of multiple angiogenic pathways using anti-angiogenics. Due to its inherent genomic heterogeneity, molecularly defined subgroups of HGSOC ("differentiated", "immunoreactive", "mesenchymal" and "proliferative") may require different approaches.

BRCA1/2 mutated OCs and OCs with a BRCAness phenotype have demonstrated sensitivity to PARP inhibitors due to underlying deficiencies in DNA homologous recombination; however, clinical responses are often partial and highly dependent on platinum sensitivity. PARP inhibitors such as olaparib, niraparib and rucaparib are already approved for treatment of recurrent EOC and their indications are partially overlapping: niraparib [37] and olaparib [38] have been approved for maintenance therapy after partial or complete remission in recurrent ovarian cancer. Further, olaparib [39] and rucaparib [40] have been approved as monotherapy for advanced recurrent OC. More recently, olaparib has demonstrated impressive activity in BRCA-mutated OC as maintenance following first-line chemotherapy (SOLO-1 trial; HR for disease progression or death, $0.30 ; 95 \% \mathrm{CI}, 0.23$ to $0.41 ; P<0.001)$. Today, probably another promising therapeutic approach in this context is the blockade of immune checkpoints, such as programmed cell death 1 (PD-1), its ligand PD-L1 or cytotoxic T-lymphocyte associated protein 4 (CTLA4), which demonstrated impressive response rates in malignant melanoma and non-small-cell lung carcinoma (NSCLC). Considering this and a positive expression of check point molecules in OC which is associated with clinical outcome [41] many clinical studies investigate check point inhibitors in OC, especially platinum-resistant or recurrent OC [42]. Various simultaneous anti-angiogenics may improve the therapeutic benefit and counteract compensatory escape mechanisms [43]. Additional studies are necessary to determine optimal combinations that could be either vertical (e.g., bevacizumab with other angiogenesis inhibitors like sorafenib, vandetanib, sunitinib), horizontal (e.g., inhibitors of phosphatidylinositol-4,5bisphosphate 3-kinase [PI3K] pathway, MAP kinseERK kinase [MEK], angiopoietin), or direct (e.g., bevacizumab with thrombospondin-1 or vascular disrupting agents such as combretastatin Al phosphate [OXi4503]) [11].

Collectively, resistance to chemotherapy and antiangiogenic approaches depends on multiple factors that are challenging to control in a clinical setting until today. Insights into tumour biology and the tumour microenvironment may help to overcome mechanisms of resistance to tackle OC progression. Increasing availability of novel (mechanistically distinct) treatment approaches in OC and the selection of patients that benefit from particular treatment modalities may improve OC survival. Addressing these issues will require further clinical investigations and identification of predictive biomarkers. 
Funding Open access funding provided by University of Innsbruck and Medical University of Innsbruck.

Conflict of interest V. Wieser and C. Marth declare that they have no competing interests.

Open Access This article is distributed under the terms of the Creative Commons Attribution 4.0 International License (http://creativecommons.org/licenses/by/4.0/), which permits unrestricted use, distribution, and reproduction in any medium, provided you give appropriate credit to the original author(s) and the source, provide a link to the Creative Commons license, and indicate if changes were made.

Publisher's Note Springer Nature remains neutral with regard to jurisdictional claims in published maps and institutional affiliations.

\section{References}

1. Ozols RF, Bundy BN, Greer BE, et al. Phase III trial of carboplatin and paclitaxel compared with cisplatin and paclitaxel in patients with optimally resected stage III ovarian cancer: a Gynecologic Oncology Group study. J Clin Oncol. 2003;21:3194-200.

2. Burger RA, Brady MF, Bookman MA, et al. Incorporation of bevacizumab in the primary treatment of ovarian cancer. NEngl J Med. 2011;365:2473-83.

3. Rubin SC, Randall TC, Armstrong KA, Chi DS, Hoskins WJ. Ten-year follow-up of ovarian cancer patients after secondlook laparotomy with negative findings. Obstet Gynecol. 1999;93:21-4.

4. Kim A, Ueda Y, Naka T, Enomoto T. Therapeutic strategies in epithelial ovarian cancer. J Exp Clin Cancer Res. 2012;31:14.

5. Davis A, Tinker AV, Friedlander M. "Platinum resistant" ovarian cancer: what is it, who to treat and how to measure benefit? GynecolOncol. 2014;133:624-31.

6. Gavalas NG, Liontos M, Trachana SP, et al. Angiogenesisrelated pathways in the pathogenesis of ovarian cancer. IntJ MolSci. 2013;14:15885-909.

7. BoocockCA, Charnock-Jones DS, SharkeyAM, etal. Expression of vascular endothelial growth factor and its receptors flt and KDR in ovarian carcinoma. J Natl Cancer Inst. 1995;87:506-16.

8. Reynolds K, Farzaneh F, Collins WP, et al. Association of ovarian malignancy with expression of platelet-derived endothelial cell growth factor. J Natl Cancer Inst. 1994;86:1234-8.

9. Aghajanian C, Goff B, Nycum LR, Wang YV, Husain A, Blank SV. Final overall survival and safety analysis of OCEANS, a phase 3 trial of chemotherapy with or without bevacizumab in patients with platinum-sensitive recurrent ovarian cancer. Gynecol Oncol. 2015;139:10-6.

10. Pujade-Lauraine E, Hilpert F, Weber B, et al. Bevacizumab combined with chemotherapy for platinum-resistant recurrent ovarian cancer: the AURELIA open-label randomized phase III trial. JClin Oncol. 2014;32:1302-8.

11. Yang X, Shen F, Hu W, Coleman RL, Sood AK. New ways to successfully target tumor vasculature in ovarian cancer. Curr Opin Obstet Gynecol. 2015;27:58-65.

12. Agarwal R, Kaye SB. Ovarian cancer: strategies for overcoming resistance to chemotherapy. Nat Rev Cancer. 2003;3:502-16.

13. Ricci F, Affatato R, Carrassa L, Damia G. Recent insights into mucinous ovarian carcinoma. Int J Mol Sci. 2018;19 https:// doi.org/10.3390/ijms19061569.
14. Alsop K, Fereday S, Meldrum C, et al. BRCA mutation frequency and patterns of treatment response in BRCA mutation-positive women with ovarian cancer: a report from the Australian Ovarian Cancer Study Group. J Clin Oncol. 2012;30:2654-63.

15. David BY, Chetrit A, Hirsh-Yechezkel G, et al. Effect of BRCA mutations on the length of survival in epithelial ovarian tumors. JClin Oncol. 2002;20:463-6.

16. Tan DS, Rothermundt C, Thomas K, et al. "BRCAness" syndrome in ovarian cancer: a case-control study describing the clinical features and outcome of patients with epithelial ovarian cancer associated with BRCA1 and BRCA2 mutations. JClin Oncol. 2008;26:5530-6.

17. Vencken PM, Kriege M, Hoogwerf D, et al. Chemosensitivity and outcome of BRCA1- and BRCA2-associated ovarian cancer patients after first-line chemotherapy compared with sporadic ovarian cancer patients. Ann Oncol. 2011;22:1346-52.

18. Lord CJ, Ashworth A. BRCAness revisited. Nat Rev Cancer. 2016;16:110-20.

19. Konstantinopoulos PA, Spentzos D, Karlan BY, et al. Gene expression profile of BRCAness that correlates with responsiveness to chemotherapy and with outcome in patients with epithelial ovarian cancer. J Clin Oncol. 2010;28:3555-61.

20. Boesch M, Sopper S, Zeimet AG, et al. Heterogeneity of cancer stem cells: rationale for targeting the stem cell niche. Biochim Biophys Acta. 2016;1866:276-89.

21. Zeimet AG, Reimer D, Sopper S, et al. Ovarian cancer stem cells. Neoplasma. 2012;59:747-55.

22. Kryczek I, Liu S, Roh M, et al. Expression of aldehyde dehydrogenase and CD133 defines ovarian cancer stem cells. Int JCancer. 2012;130:29-39.

23. Silva IA, Bai S, McLean K, et al. Aldehyde dehydrogenase in combination with $\mathrm{CD} 133$ defines angiogenic ovarian cancer stem cells that portend poor patient survival. Cancer Res. 2011;71:3991-4001.

24. Landen CN Jr., Goodman B, Katre AA, et al. Targeting aldehyde dehydrogenase cancer stem cells in ovarian cancer. MolCancerTher. 2010;9:3186-99.

25. Reimer D, Boesch M, Wolf D, et al. Truncated isoform Vav3.1 is highly expressed in ovarian cancer stem cells and clinically relevant in predicting prognosis and platinumresponse. Int JCancer. 2018;142:1640-51.

26. Zahreddine H, Borden KL. Mechanisms and insights into drug resistance in cancer. Front Pharmacol. 2013;4:28.

27. Tomao F, Papa A, Rossi L, et al. Beyond bevacizumab: investigatingnewangiogenesisinhibitorsin ovariancancer. Expert Opin Investig Drugs. 2014;23:37-53.

28. Winiarski BK, Wolanska KI, Rai S, et al. Epithelial ovarian cancer-induced angiogenic phenotype of human omental microvascular endothelial cells may occur independently of VEGF signaling. Transl Oncol. 2013;6:703-14.

29. Noy R, Pollard JW. Tumor-associated macrophages: from mechanisms to therapy. Immunity. 2014;41:49-61.

30. Baeriswyl V, Christofori G. The angiogenic switch in carcinogenesis. Semin Cancer Biol. 2009;19:329-37.

31. WangX, ZhaoX, Wang K, Wu L, Duan T. Interaction of monocytes/macrophages with ovarian cancer cells promotes angiogenesis in vitro. Cancer Sci. 2013;104:516-23.

32. Welsh JB, Zarrinkar PP, Sapinoso LM, et al. Analysis of gene expression profiles in normal and neoplastic ovarian tissue samples identifies candidate molecular markers of epithelial ovarian cancer. Proc Natl Acad Sci U S A. 2001;98:1176-81.

33. Iorio MV, Visone R, Di Leva G, et al. MicroRNA signatures in human ovarian cancer. Cancer Res. 2007;67:8699-707. 
34. Nam EJ, Yoon H, Kim SW, et al. MicroRNA expression profiles in serous ovarian carcinoma. Clin Cancer Res. 2008;14:2690-5.

35. Kommoss S, Winterhoff B, Oberg AL, et al. Bevacizumab May differentially improve ovarian cancer outcome in patients with proliferative and mesenchymal molecular subtypes. Clin Cancer Res. 2017;23:3794-801.

36. Colombo PE, Fabbro M, Theillet C, Bibeau F, RouanetP, RayCoquard I. Sensitivity and resistance to treatment in the primary management of epithelial ovarian cancer. Crit Rev Oncol Hematol. 2014;89:207-16.

37. Mirza MR, Monk BJ, Herrstedt J, et al. Niraparib maintenance therapy in platinum-sensitive, recurrent ovarian cancer. NEngl J Med. 2016;375:2154-64.

38. Pujade-Lauraine E, Ledermann JA, Selle F, et al. Olaparib tablets as maintenance therapy in patients with platinum-sensitive, relapsed ovarian cancer and a BRCA1/2 mutation (SOLO2/ENGOT-Ov21): a double-blind, randomised, placebo-controlled, phase 3 trial. Lancet Oncol. 2017;18:1274-84.

39. Matulonis UA, Penson RT, Domchek SM, et al. Olaparib monotherapy in patients with advanced relapsed ovarian cancer and a germline BRCA1/2 mutation: a multistudy analysis of response rates and safety. Ann Oncol. 2016;27:1013-9.
40. Balasubramaniam S, Beaver JA, Horton S, et al. FDA approval summary: rucaparib for the treatment of patients with deleterious BRCA mutation-associated advanced ovarian cancer. Clin Cancer Res. 2017;23:7165-70.

41. Wieser V, Gaugg I, Fleischer M, et al. BRCA1/2 and TP53 mutation status associates with PD-1 and PD-L1 expression in ovarian cancer. Oncotarget. 2018;9:17501-11.

42. Marth C, Wieser V, Tsibulak I, Zeimet AG. Immunotherapy in ovarian cancer: fake news or the real deal? International Journal of Gynecologic Cancer. 2019;29(1):201-11.

43. Moreno Garcia V, Basu B, Molife LR, Kaye SB. Combining antiangiogenics to overcome resistance: rationale and clinical experience. Clin Cancer Res. 2012;18:3750-61.

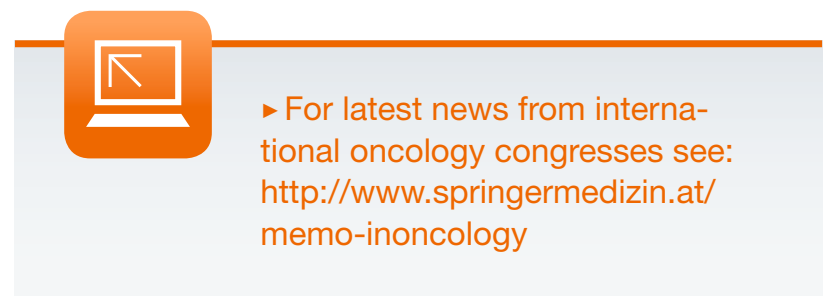

